\title{
The Analysis of Face Recognition Based on BP Artificial Neural Network
}

$$
\text { Yang } \mathrm{Du}^{1, \mathrm{a}} \text {, Fei Guo }{ }^{\mathrm{b}^{*}}
$$

${ }^{1}$ School of Information Engineering, Beijing Institute of Fashion Technology, Beijing 100029, China

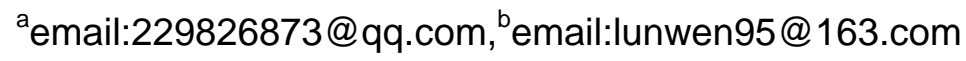

Keywords: Artificial neural network, face recognition, pattern recognition, computer technology

\begin{abstract}
Today, with the constant development of science and technology, computer technology is improved gradually as well. But in today's computer technology development, the part of face recognition became a hot research point in pattern recognition of computer with the vast development prospect and application. Face can be recognized by Human's brain accurately, because there is complex neural network in human's brain. So at the same time of computer development, Similar to the human's brain neural network, also the artificial neural network is being developed constantly. In the paper, based on BP artificial neural network the face recognition is introduced, and it is complemented through introducing the model of BP neural network.
\end{abstract}

\section{Introduction}

The face recognition in today's science and technology is became more and more active, and with many requirements a variety of function has been found in face recognition. As the most significant part of the feature in human's external recognition aspect[1], and for human's recognition face recognition has its important meaning. Composed with fingerprint and iris recognition, human's face recognition could be more hard because of factors of environmental conditions, such as light and bearing.

The feature's extracted for human's eyes, nose and mouse is identified as main technology aspect of face recognition in the paper, and then starting face recognition. In the function of face recognition, for its speed, recognition rate and fault tolerance, there is quite a good advantage in $\mathrm{BP}[2]$ artificial neural network, and more complex feature extracted is avoided effectively. Through some primary feature extracted, and learning and comparison is processed again and again, so face recognition can be achieved.

\section{BP Artificial Neural Network}

\section{Neural Network}

\section{Biological Network}

For learning artificial neural network ${ }^{[4]}$, the neurons and network of human 's brain must be known. Neural network in human's brain is constituted of plenty of neurons. Just for these neurons, complex studying, feeling and living can be done for human. For one neuron in neural network, its structure is shown in figure1:

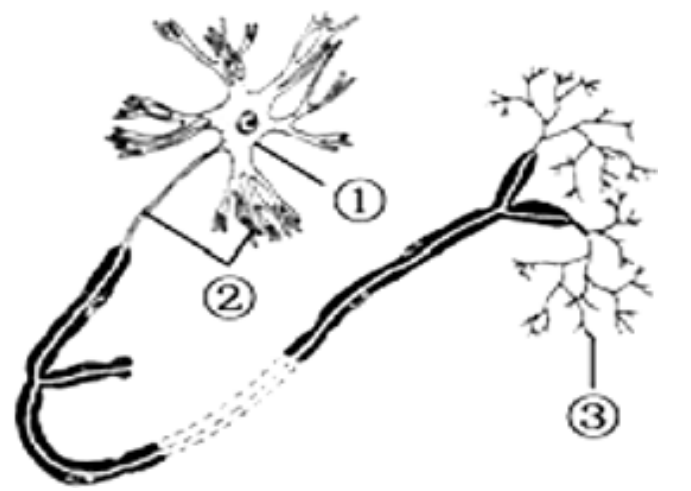

Fig.1. (1)cell body (2)dendrite (3)synapsis 
Through simply dealt information of coming from interior or exterior by Biological neurons, then, the processed signal would be send out. Biological neural network is made up of much more neurons with linked. Through plenty of simple neurons complex linking modus, ample way of act would be generated by biology. But, because of unstable linking among neurons, in the biological studying, some new connection generated and also some will disappear. External stimuli called the input of neural network, this whole network would be stimulated the signal, each neuron adjusts the strength of their connection until being stable.

\section{Artificial Neural Network}

Above this paper, biological neural network is composed by neurons, so artificial neural network is made up of artificial neurons as well. And it includes three important part: Input terminal(Dendrite), processing terminal(Cell Body), output terminal (Synapsis). Its simple model has been displayed as figure2:

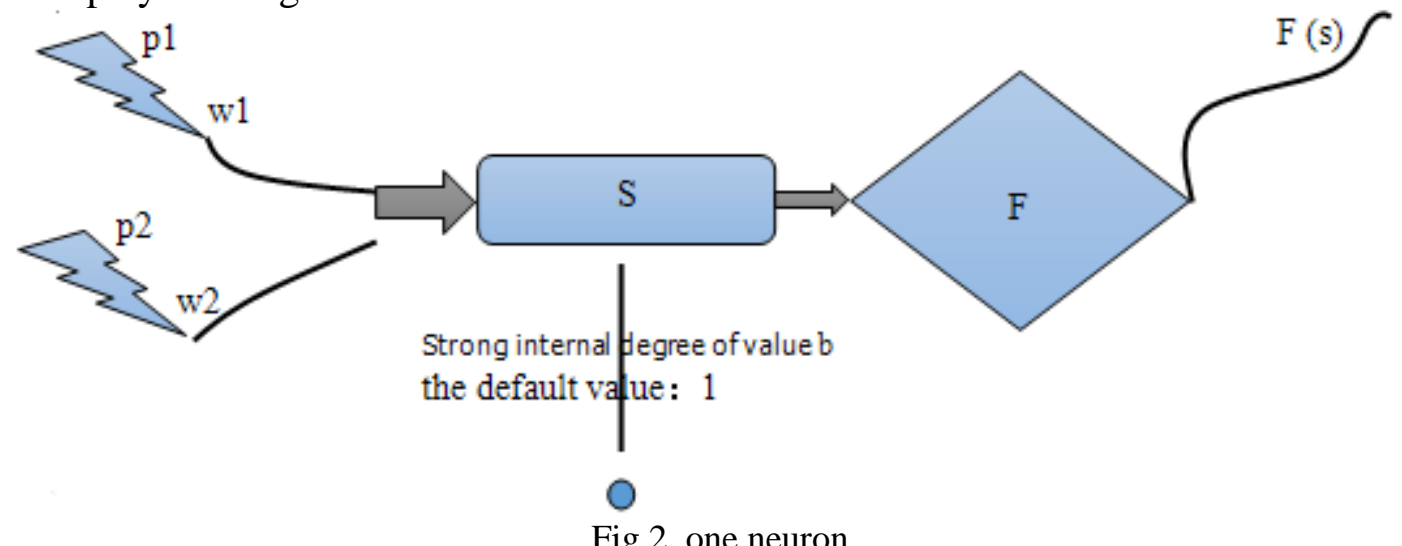

Fig.2. one neuron

In the figure2, p1 and p2 is represented by input(Dendrite), w1 and w2 stands for the weighted value of input, $s$ represents processed signal, $f$ delegates transfer function, $f(s)$ typifies output signal of output terminal. There, s could be represented by formula(1):

$\mathrm{s}=\mathrm{p} 1 * \mathrm{w} 1+\mathrm{p} 2 * \mathrm{w} 2+\mathrm{b} * 1$

If an artificial neuron includes many input terminal, the relative formula is as formula(2):

$\mathrm{s}=\sum_{i=1}^{\mathrm{n}} p i * w i+b * 1$

Therefore, single artificial neuron has been simulated completely. The same as biological neural network, artificial neural network is also made up of many artificial neurons' connection.

\section{BP Artificial Neural Network}

BP neural network has three parts: input layer, hidden layer and output layer ${ }^{[5]}$. It is a feedback type neural network which means error back propagation learning, and it has been used widely. Its full name called Back Propagation, also called Error Back Propagation. Its main idea means: compared real output value with prospective output, in the existing error situation, error would be transfer from inverted direction output, it will be allotted to each connection of weighted value between neurons, then every neuron would receive the error signal, recognizing this signal as basis of revising weighted value. Through these studying back and forth, until the network satisfied the relationship.

Face recognition is a processing which is divided based on eigenvector, that's called classifier. Using BP neural network to design face recognition of classifier, the reason why use it is not only that this arithmetic is relatively simple and mellow, but also the face eigenvector becomes lower. For these, BP model will be meliorated in training time. So one ordinary BP neural network model has been revealed as figure3: 


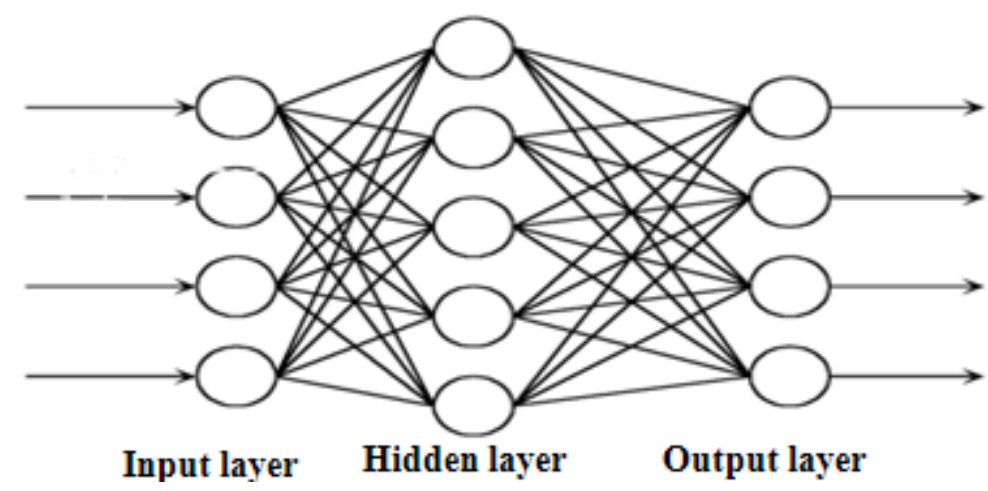

Fig.3. BP neural network model

The studying regulation of artificial neural network:

$$
\begin{aligned}
& w(\text { new })=w(\text { old })+e * p \\
& b(\text { new })=w(\text { old })+e
\end{aligned}
$$

In these formula, $\mathrm{e}$ is error, $\mathrm{e}=\mathrm{t}-\mathrm{a}, \mathrm{t}$ represented prospective output value, a stands for real output. Through this studying processing, the whole neural network will be stable.

\section{BP Arithmetic in Neural Network}

\section{BP Neural Network Arithmetic \\ BP Network}

Three layers make up of BP neural network, and neuron weight value connects adjacent layer, same layer exist no neuron. For the input and output layer of BP network, they are the same, called $X_{j}=O_{i}$. The relationship of hidden layer's input and input layer's output is as fallowing:

$$
\begin{gathered}
\operatorname{Net}_{i}=\sum W_{j i} * O_{i} \\
O_{j}=\mathrm{f}\left(\text { Net }_{j}\right)
\end{gathered}
$$

In these formula, $\mathrm{Net}_{j}$ represents that neuron $\mathrm{j}$ receive total input. $W_{j i}$ stands for the weight value from forth layer's neuron i to this layer's neuron $j$. And sigmoid function is generally used to f: $\mathrm{f}(\mathrm{x})=1 /\left(1+e^{-x}\right)$, its graph of function is as fallowing:

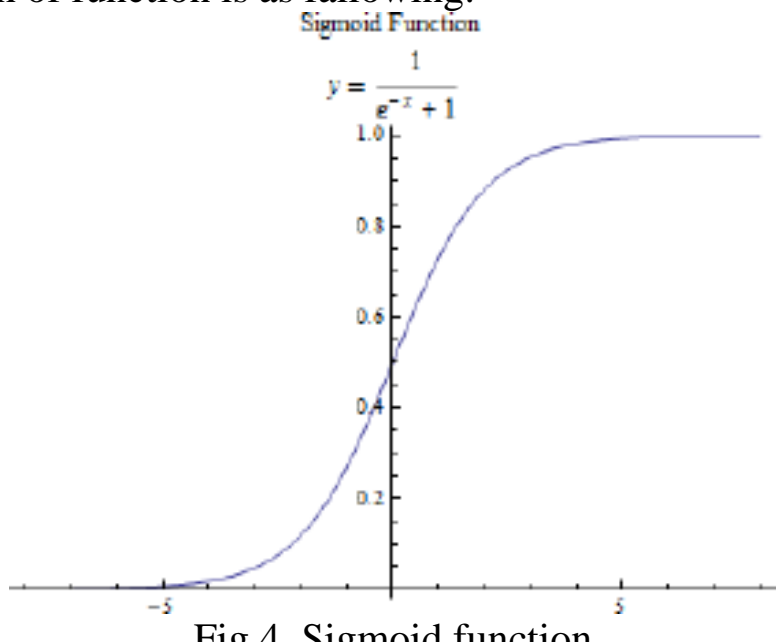

So, $O_{j}$ is generated by $\mathrm{Net}_{j}$ through f function.

\section{Selecting the Number of Neurons}

Through the extraction of input layer model's feature between hidden layer and input layer's weight value is widely regarded as BP neural network, and then, sending these feature to output layer. In the whole BP neural network, the number of input layer's neurons equals I, and hidden layer is $\mathrm{H}$, $\mathrm{J}$ represents output layer. For the choice of $\mathrm{H}$, if $\mathrm{H}$ is lager, the time of network's training 
would be increased. And for concerning the classifier's stability, the quantity of network could not over the training sample size, so the choice of $\mathrm{H}$ as fallowing:

\section{$K * I \geq(\mathrm{H}+1) * J+(\mathrm{I}+1) * \mathrm{H}$}

From the formula(4), knowing the amount of neurons and training number $\mathrm{K}$ between input layer and output layer, the neurons' quantity would be worked out.

\section{BP Arithmetic Process Neural Network}

In the neural network, the sigmoid function is regarded as transfer function and counter propagation function. So this arithmetic's procedure as fallowing:

(1) Setting relevant variable and parameter.

(2) Initialize.

(3) Working out output quantity of each neuron towards counter.

(4) Figuring out final error of output layer.

(5) Computing the error of hidden layer.

(6) Calculation of each neuron's weight value.

$\Delta W_{i j}(t)=\alpha * \Delta W_{i j}(t-1)+\eta \delta_{j} O_{j}$

(7) Revising the weight value.

$W_{i j}(t+1)=W_{i j}(t)+\Delta W_{i j}(t)$

Through fallowing steps, a simple BP neural network can do the training learning.

\section{Achievement of BP Artificial Neural Network in Face Recognition}

On the BP neural network face recognition, the first thing is to extract eigenvalue of face to be matrix, and work out the feature vectors $\mathrm{T}$ and descending order, then choosing the large characteristics of root N. Then, sending the feature vectors to BP neural network. After that, this feature vectors do learning and training according to the rule of neural network. After training, that would be sent to output layer, and comparing expected value with real value, then working out the error value. Afterwards, in order to share this error to each neuron it would be sent to output layer in the whole network. Through this model to training and learning again and again, until the real value and expected value could be matched, then the procession of face recognition is done well.

There, BP neural network arithmetic is used reversely to encode and achieve in MATLAB, for this, importing relevant test image to train and learn.

Firstly, doing face detection, and 'lenna.jpg' is used to test image. Its effect as fallowing:

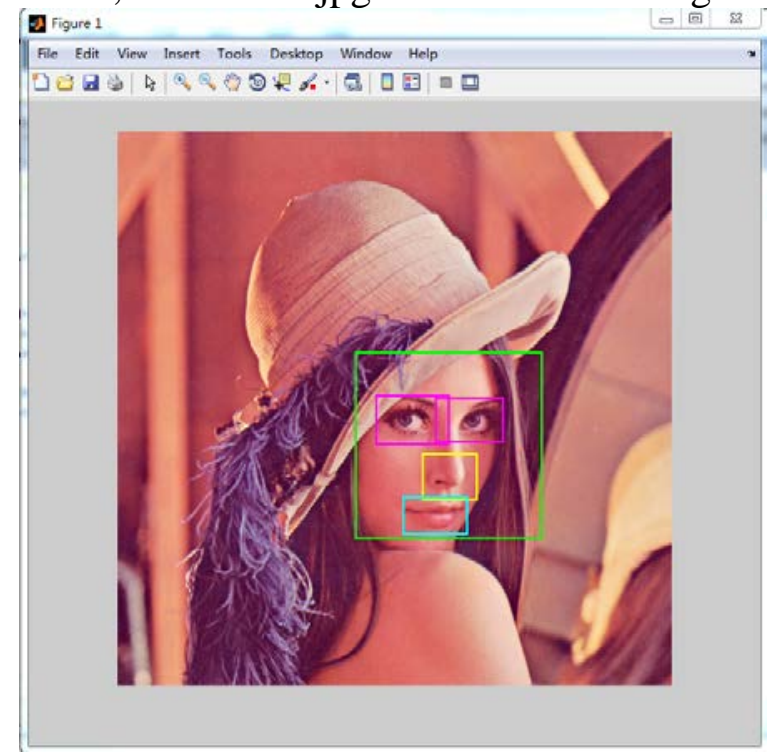

Fig.5. Face Detection

From above image, after extract of feature value for eyes, nose and mouth, the detection of face is indicated.

Then, the next step is that using BP neural network achieves the face detection. There are two libraries, one called TrainDatabase, the others called TestDatabase. The TrainDatabase library exist 
many face images, TestDatabase library exist relevant face image with TrainDatabase library. The achievement of BP neural network arithmetic is doing training and learning for face in MATLAB, and then, TestDatabase library would do face detection. There, according relevant arithmetic, after operation step by step, this effect is as fallowing:
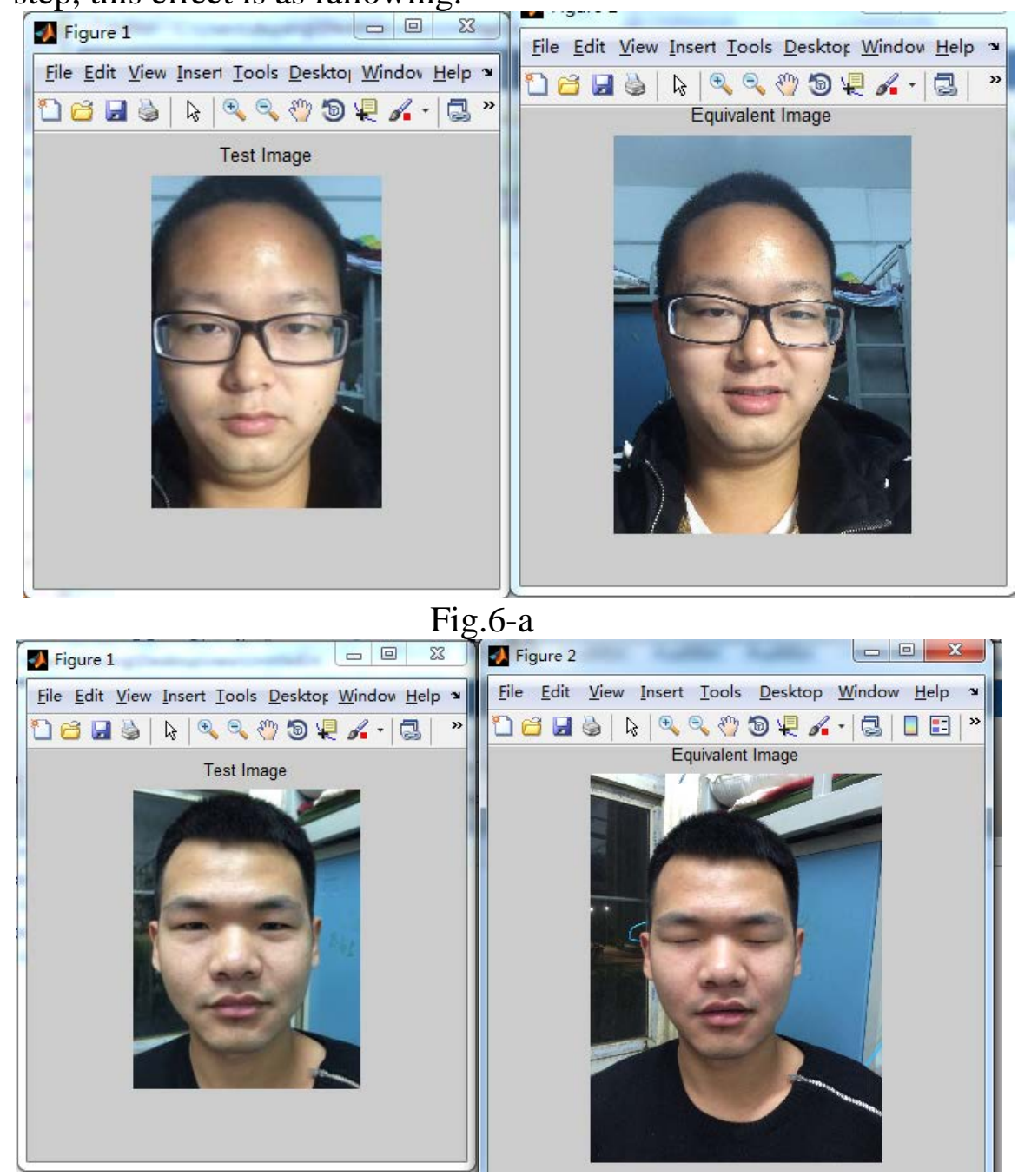

Fig.6-b

Fig.6. the test image of BP neural network arithmetic

From these figures, Equivalent Image has been obtained by the Test Image through BP artificial neural network. So, BP artificial neural network has high accuracy in face recognition.

\section{Conclusion}

Face recognition has been used in too much area, especially in some security protection and some especial department. At the present time, the measures of face recognition is too much more. So in these measures, a suitable measure must be researched to achieve the requirement of face recognition.

Therefore, as described BP neural network arithmetic in this paper, its accuracy and efficiency of recognition time both has preferable advantages. Combined with the model of self-organization of artificial neural network learning and BP arithmetic, efficiency and accuracy of face recognition would be improved highly at the learning and training. So, BP artificial neural network would be used more and more widely.

\section{Acknowledgement}

In this paper, the research was sponsored by the Innovation Team from Project of Beijing institute of fashion technology (Project No. NHFZ2016044/008) and Promoting the Fixed Special 
Connotation and Development in Colleges and Universities of Beijing institute of fashion technology (Project No. NHFZ2016012) and Beijing university project steering committee 'University library reader recommendation resources evaluation research' (Project No. BGT2016026) and Beijing municipal education commission on the project of Based on the user driven recommendation platform construction of university library resources research (K Y J H 02 $160201 / 002 / 022)$.

\section{References}

[1] S.L.Xie, Y.H.Zhang, C.H.Chen,X.N.Zhang. Identification of nonlinear hysteretic systems by artificial neural network [J]. Mechanical Systems and Signal Processing.34(2013)76-87.

[2] C.Zhang, W.Wu,X.H.Chen, Y.Xiong. Convergence of BP algorithm for product unit neural networks with exponential weights[J]. Neurocomputing.2008, 12, 72:513-520.

[3] Yan Pingfan.Some view son the research of multilayer feed forward neural networks, ACTA Automatic Sinica(in Chinese), 1997,23(1): 129-135.

[4] Alain Yee-Loong Chong.Predicting m-commerce adoption determinants: A neural network approach[J].Expert Systems with Applications 40 (2013) 523-530.

[5] Xiangyang Zhao, Kangsheng Lai, Dongming Dai. An improved BP Algorithm and Its Application in Classification of Surface Defects of Steel Plate[J]. Journal of iron and steel research,international, (2007), 14(2):52-55

[6] C.Zhang, W.Wu, X.H.Chen, Y.Xiong Convergence of BP algorithm for product unit neural network with exponentional weights[J]. Neurocomputing 2008, 72(1):513-520.

[7] D.E.Rumelhart, G.E.Hinton, R.J.Williams. Learning internal representations by error propagation[C].Parallel distributed processing, Cambridge,MA:The MIT Press,1986,(1):318-362. 